\section{Fatores associados à inatividade física no lazer e principais barreiras na percepção de trabalhadores da indústria do Sul do Brasil}

\author{
Variables associated with leisure-time physical \\ inactivity and main barriers to exercise among \\ industrial workers in Southern Brazil
}

\author{
1 Programa de Pós-graduação \\ em Educação Física, \\ Universidade Federal de \\ Pelotas, Pelotas, Brasil. \\ 2 Programa de Pós- \\ graduação em Educação \\ Física, Universidade \\ Federal de Santa Catarina, \\ Florianópolis, Brasil. \\ 3 Serviço Social da Indústria, \\ Porto Alegre, Brasil. \\ Correspondência \\ M. C. Silva \\ Programa de Pós-graduação \\ em Educação Física, \\ Universidade Federal de \\ Pelotas. \\ Rua Luis de Camões 625, \\ Pelotas, RS 96055-360, Brasil. \\ cozzensa@terra.com.br
}

\section{Abstract}

The aim of this study was to identify the main perceived barriers to leisure-time physical activity and factors associated with physical inactivity among industrial workers in the State of Rio Grande do Sul, Brazil. A cross-sectional study with analysis of secondary data included 2,265 workers. Thirteen barriers were investigated: fatigue, weather, overwork, lack of will, study obligations, family obligations, distance to the facility/setting, affordability, lack of motor skills, poor physical conditioning, lack of facilities, lack of money, security, and others. Prevalence of physical inactivity among workers was $45.4 \%$, and the most common barriers were fatigue (15.1\%), overwork (12.7\%), and family obligations (9.2\%). Fatigue, overwork, family obligations, lack of will, affordability, study obligations, and weather were associated with leisure-time physical inactivity. Government policies focused on overarching interventions could help these workers overcome such barriers and adopt an active lifestyle.

Motor Activity; Leisure Activities; Occupational Groups
Shana Ginar da Silva ${ }^{1}$

Marcelo Cozzensa da Silva 1

Markus Vinícius Nahas 2

Sérgio Luís Viana 3

\section{Introdução}

O local de trabalho se configura como um importante espaço para o desenvolvimento de intervenções visando à promoção da saúde e qualidade de vida, visto a possibilidade de atingir um grande número de pessoas e porque grande parte da população adulta destina inúmeras horas de suas vidas ao labor 1 .

Quando nos referimos à saúde do trabalhador, baixos índices de aptidão física podem predispor a distúrbios ocupacionais, tais como lesão por esforço repetitivo e doenças osteomusculares relacionadas ao trabalho, os quais são caracterizados por sensação de cansaço, tensão muscular e dor postural, entre outros sintomas. Além disso, baixos índices de aptidão física podem impactar negativamente na saúde mental e relações sociais do trabalhador 2 .

Vários são os determinantes que podem influenciar a participação em atividades físicas de lazer. Um estilo de vida fisicamente ativo pode ser determinado por características socioeconômicas, demográficas, comportamentais e ambientais, bem como pelo nível de conhecimento, atitudes e crenças acerca das atividades físicas 3 .

Esses determinantes, em algumas situações, podem ser facilitadores do comportamento ativo, em outras podem ser percebidas como barreiras à prática de atividades físicas 4,5 . 
As evidências científicas apontam que, quanto maior a faixa etária estudada, maior a prevalência de sedentarismo, e mulheres tendem a ser mais sedentárias no lazer. Por outro lado, quando a avaliação é realizada nos quatro domínios (lazer, deslocamento, trabalho e doméstico), as prevalências entre os sexos são muito próximas 6 . Nível socioeconômico e escolaridade são diretamente proporcionais à inserção em atividades físicas no lazer. Já em análises dos quatro domínios, essa relação passa a ser inversa 7 . Além disso, outros fatores estão associados ao nível de atividade física, como situação conjugal, obesidade, tabagismo, falta de tempo, hábito de exercício no passado, entre outros ${ }^{8}$.

A participação em atividades físicas também pode ser positivamente ou negativamente influenciada pela percepção de barreiras. Falta de tempo, falta de dinheiro, falta de motivação, sentir-se cansado, clima, são algumas das barreiras mais prevalentes reportadas pela população à prática de atividade física no lazer 9,10,11.

Em países desenvolvidos, a falta de tempo é muito reportada como barreira e pode ser fomentada pela falta de motivação ${ }^{12}$. Em contrapartida, a dificuldade financeira é pouco relatada 9 . Já em países em desenvolvimento, com realidades opostas, outros tipos de limitações são apresentados frequentemente, sendo maior a prevalência dessas barreiras 10 .

Um estudo realizado com adultos em 15 países europeus demonstrou que o trabalho e os compromissos com os estudos são fortemente apontados como barreiras à prática de atividades físicas. Além disso, o estudo conclui que a percepção de barreiras e a magnitude da associação com o nível de atividade física dependem da população estudada ${ }^{13}$. Nesse contexto, os trabalhadores parecem estar expostos a barreiras específicas em relação à adoção de comportamentos mais saudáveis 14. Contudo, em âmbito nacional, poucas pesquisas têm focado suas investigações na distribuição dos determinantes e barreiras deste grupo populacional.

Portanto, os objetivos do presente estudo foram identificar as principais barreiras percebidas à prática de atividades físicas no lazer e verificar quais são os fatores associados à inatividade física no lazer em trabalhadores industriários do Estado do Rio Grande do Sul, Brasil. Tais dados, escassos na literatura que estuda trabalhadores, darão suporte à criação de estratégias de intervenção para a modificação dessa realidade.

\section{Métodos}

Este estudo caracteriza-se por ser do tipo observacional de corte transversal com análise secundária de dados. Utilizou-se uma amostra representativa dos 670.326 trabalhadores da indústria do Estado do Rio Grande do Sul participantes do estudo epidemiológico transversal Perfil do Estilo de Vida e Hábitos de Lazer de Trabalhadores da Indústria do Estado do Rio Grande do Sul/Brasil, realizado no período de agosto a dezembro de 2007. Este estudo resultou de uma parceria do SESI/DN (Serviço Social da Indústria, Departamento Nacional) com o Núcleo de Pesquisa em Atividade Física e Saúde da Universidade Federal de Santa Catarina (NuPAF/UFSC). A amostra foi determinada em dois estágios, de modo a ser representativa do conjunto de indústrias das oito unidades regionais classificadas pelo SESI/DRRS (Serviço Social da Indústria, Departamento Regional do Rio Grande do Sul) 15. O tamanho mínimo da amostra foi calculado considerando um erro amostral de 3\%, um intervalo de $95 \%$ de confiança (IC95\%), uma prevalência de inatividade física no lazer de $45 \%$, tendo-se acrescentado $50 \%$ a este número em função do delineamento amostral (efeito do delineamento amostral $=1,5$ ) e mais $20 \%$ em função das possíveis perdas no processo de coleta 16 .

No primeiro estágio, recorreu-se à seleção aleatória de empresas tendo em conta a distribuição dos trabalhadores em empresas de grande (500 ou mais trabalhadores), médio (100 a 499) e pequeno porte (20 a 99). Foram selecionadas aleatoriamente 20 a $50 \%$ das empresas de grande, médio e pequeno porte a depender do número de empresas existentes e do número requerido de trabalhadores para composição da amostra. No segundo estágio, de cada uma das empresas sorteadas na fase anterior do processo amostral foram selecionados, também de forma aleatória, trabalhadores de ambos os sexos em número proporcional ao porte da empresa.

Estimando-se uma prevalência de inatividade física nos não expostos em torno de $30 \%$ e a relação exposto/não exposto variando de 1:99 (barreira - falta de instalações), a amostra estudada ( $\mathrm{n}=2.265$ ) confere um poder estatístico superior a $80 \%$ para examinar associações com um risco relativo em torno de 2,0 e um nível de 95\% de confiança.

As empresas que não permitiram a aplicação dos questionários foram substituídas por empresas do mesmo porte e preferencialmente do mesmo ramo. Para substituição daquele trabalhador selecionado que não estava no local de trabalho no momento da coleta ou que se negasse a participar, procedeu-se à escolha do nome imedia- 
tamente posterior na relação de empregados. Optou-se por essas duas estratégias de reposição para minimizar as perdas amostrais em determinadas regiões com pequena quantidade de indústrias, o que poderia inviabilizar o estudo pela falta de poder e representatividade.

A coordenação do estudo foi realizada por uma equipe formada entre o NuPAF/UFSC e por um representante da área de lazer do SESI/DRRS, que tinha como função supervisionar o trabalho de campo realizado pelos gestores e fazer o contato direto com a equipe do NuPAF/UFSC.

Para garantir a padronização da coleta de dados, trinta (30) gestores locais (representantes das oito unidades regionais classificadas pelo do SESI/DR-RS) foram treinados por uma equipe do NuPAF/UFSC, valendo-se de uma videoconferência que objetiva a padronização dos procedimentos de aplicação dos questionários. Em seguida, cada gestor ficou responsável pela tarefa de solicitar a autorização das empresas da sua região para realização do levantamento, providenciar a listagem para realizar o sorteio dos trabalhadores e treinar a sua equipe (profissionais do SESI e prestadores de serviço) para a realização da coleta de dados.

A coleta foi realizada em pequenos grupos (3 a 15 trabalhadores) pelos profissionais do SESI e prestadores de serviço, que participaram de treinamento específico para padronização da aplicação dos questionários. Os gestores fizeram contato prévio formal com a administração das empresas selecionadas para marcação do dia e local para coleta. Todos os trabalhadores foram informados do caráter voluntário da participação no levantamento e do sigilo de suas respostas, sem identificação dos respondentes.

No levantamento das informações, usou-se um questionário previamente validado e originalmente construído para tal população 17 .

O desfecho percepção de barreiras foi mensurado por questão fechada com 13 diferentes opções de resposta (cansaço, clima, excesso de trabalho, falta de vontade, obrigações de estudo, obrigações familiares, distância até o local da prática, falta de habilidade motora, falta de condições físicas, falta de instalações, falta de dinheiro e condições de segurança, além da opção outra), mas o trabalhador poderia apontar apenas uma barreira (Figura 1). Já o desfecho prática de atividade física no lazer foi mensurado com base na questão "Você realiza regularmente, algum tipo de atividade física no seu lazer, como: exercícios físicos (ginástica, caminhada, corrida), esportes, danças ou artes marciais?". Com cinco opções de resposta (sim, 1 ou 2 vezes por semana; sim, 3 ou 4 vezes por semana; sim, 5 ou mais vezes por semana; não, mas estou interessado(a) em realizar atividade física no meu lazer num futuro próximo; e não estou interessado(a) em realizar atividade física no meu lazer num futuro próximo) que foram subseqüentemente dicotomizadas.

Foram considerados inativos aqueles indivíduos que relataram não praticar algum tipo de atividade física por semana, e ativos aqueles que relataram realizar atividades físicas em, no mínimo, uma vez por semana.

Foram analisadas como exposições variáveis demográficas (sexo, idade, estado civil), socioeconômicas (escolaridade e renda familiar) e comportamentais (nível de estresse, fumo, consumo de bebidas alcoólicas) e de trabalho (satisfação no trabalho).

Todas as informações foram coletadas por meio de um único instrumento, contendo 58 questões agrupadas em quatro módulos: (I) informações pessoais e da indústria; (II) indicadores de saúde e comportamentos preventivos; (III) atividade física e opções de lazer; e (IV) controle do peso corporal e hábitos alimentares. No entanto, para o presente estudo, apenas as variáveis que foram descritas como desfecho e exposições foram analisadas.

As questões investigadas neste estudo com relação aos dados demográficos e socioeconômicos estavam dispostas no bloco I do questionário.

As variáveis comportamentais (nível de estresse, fumo, consumo de bebidas alcoólicas) e a variável de trabalho (satisfação no trabalho) estavam inseridas no módulo II. A variável estresse foi mensurada com base na questão: "Como você classifica o nível de estresse em sua vida?”. Com quatro opções de resposta que foram subsequentemente dicotomizadas (raramente/às vezes $=$ não; quase sempre/sempre $=$ sim). A variável fumo foi medida com base na questão: "Com relação ao fumo, qual a sua situação?”. Com seis opções de resposta: nunca fumei; parei de fumar há mais de dois anos; parei de fumar há menos de dois anos; fumo até 10 cigarros/dia; fumo de 10 a 20 cigarros/dia; fumo mais de 20 cigarros/ dia. Posteriormente, a variável fumo foi categorizada em três categorias. Já a variável consumo de bebidas alcoólicas foi mensurada pela questão: "Quantas doses de bebidas alcoólicas você toma em uma semana normal?". Quanto à satisfação no trabalho, foi formulada a seguinte questão: “Como você se sente, atualmente, quando está no trabalho?”. As descrições das categorias estão na Tabela 1.

Análises bivariadas examinaram tabelas de contingência e a associação estatística foi aferida para valor de $\mathrm{p}<0,05$, pelo teste de qui-quadrado para heterogeneidade ou tendência linear. 
Figura 1

Instrumento para mensurar barreiras à prática de atividades físicas no lazer.

\begin{tabular}{|c|c|}
\hline ( ) Cansaço & ( ) Falta de vontade \\
\hline ( ) Excesso de trabalho & ( ) Obrigações familiares \\
\hline ( ) Obrigações de estudo & ( ) Falta de habilidade motora \\
\hline ( ) Distância até o local da prática & ( ) Falta de instalações \\
\hline ( ) Falta de condições físicas (aptidão, disposição) & ( ) Outra \\
\hline ( ) Falta de dinheiro & ( ) Não tenho dificuldades \\
\hline
\end{tabular}

Nota: O instrumento na íntegra está disponível no Relatório Geral sobre Estilo de Vida e Hábitos de Lazer das Indústrias Brasileiras, 2009, na página da Internet http://www.sesi.org.br/portal/main.jsp?lumPageld=8A81818B1494CE240114983D8C E75106\&itemld=FF80808121EC48380121F36C885B5450.

Tabela 1

Características demográficas, socioeconômicas, comportamentais e de trabalho em industriários. Rio Grande do Sul, Brasil, 2007.

\begin{tabular}{|c|c|c|}
\hline Variáveis & $\mathbf{n}$ & $\%$ \\
\hline \multicolumn{3}{|l|}{ Sexo $[n=2.254]$} \\
\hline Masculino & 1.267 & 56,2 \\
\hline Feminino & 987 & 43,8 \\
\hline \multicolumn{3}{|l|}{ Idade (anos) $[\mathrm{n}=2.258]$} \\
\hline$<30$ & 1.075 & 47,6 \\
\hline $30-39$ & 651 & 28,8 \\
\hline $40-49$ & 385 & 17,1 \\
\hline $50+$ & 147 & 6,5 \\
\hline \multicolumn{3}{|l|}{ Estado civil [n = 2.261] } \\
\hline Solteiro & 1.024 & 45,3 \\
\hline Casado/Vive com companheiro & 1.237 & 54,7 \\
\hline \multicolumn{3}{|l|}{ Escolaridade [ $\mathrm{n}=2.259]$} \\
\hline Fundamental incompleto & 379 & 16,8 \\
\hline Fundamental completo & 379 & 16,8 \\
\hline Médio completo & 1.179 & 52,2 \\
\hline Superior completo & 322 & 14,2 \\
\hline \multicolumn{3}{|l|}{ Renda $(R \$)[n=2.233]$} \\
\hline Até 600 & 385 & 17,2 \\
\hline $601-1.500$ & 1.019 & 45.6 \\
\hline $1.501-3.000$ & 577 & 25,8 \\
\hline$>3.000$ & 252 & 11,4 \\
\hline \multicolumn{3}{|l|}{ Nível de estresse $[n=2.261]$} \\
\hline Raramente/Às vezes & 1.992 & 88,1 \\
\hline Quase sempre/Sempre & 269 & 11,9 \\
\hline
\end{tabular}

(continua) 


\begin{tabular}{|c|c|c|}
\hline Variáveis & n & $\%$ \\
\hline \multicolumn{3}{|l|}{ Satisfação no trabalho $[\mathrm{n}=2.262]$} \\
\hline Muito bem & 529 & 23,4 \\
\hline Bem & 1.364 & 60,3 \\
\hline Mais ou menos & 341 & 15,0 \\
\hline Mal & 24 & 1,1 \\
\hline Muito mal & 4 & 0,2 \\
\hline \multicolumn{3}{|l|}{ Fumo $[n=2.258]$} \\
\hline Nunca fumou & 1.618 & 71,6 \\
\hline Ex-fumante & 354 & 15,7 \\
\hline Atual fumante & 286 & 12,7 \\
\hline \multicolumn{3}{|c|}{ Consumo de álcool (doses/semana) [ $n=2.249]$} \\
\hline Nenhuma & 1.383 & 61,5 \\
\hline $1-7$ & 804 & 35,7 \\
\hline $8-14$ & 49 & 2,2 \\
\hline $15+$ & 13 & 0,6 \\
\hline \multicolumn{3}{|l|}{ Percepção de barreiras [n = 1.757] * } \\
\hline Cansaço & 338 & 15,1 \\
\hline Clima desfavorável & 85 & 3,8 \\
\hline Excesso de trabalho & 284 & 12,7 \\
\hline Falta de vontade & 200 & 8,9 \\
\hline Obrigações de estudo & 171 & 7,6 \\
\hline Obrigações familiares & 207 & 9,2 \\
\hline Distância até o local da prática & 70 & 3,1 \\
\hline Falta de habilidade motora & 8 & 0,4 \\
\hline Falta de condições físicas & 46 & 2,0 \\
\hline Falta de instalações & 18 & 0,8 \\
\hline Falta de dinheiro & 190 & 8,5 \\
\hline Condições de segurança & 31 & 1,4 \\
\hline Outra & 109 & 4,8 \\
\hline
\end{tabular}

* A variável percepção de barreiras foi a que apresentou maior número de missings; 475 sujeitos relataram não ter dificuldades para a prática de atividades físicas.

A análise multivariável foi realizada por regressão de Poisson, que permitiu controle simultâneo de fatores que levaram em conta a hierarquia de determinação do sedentarismo, sendo as barreiras, nessa análise, variáveis de exposição. O efeito de delineamento, com valor igual a 1,5, foi considerado na análise. O modelo proposto para a hierarquia citada foi constituído de quatro níveis: o primeiro, em que estão inseridas as variáveis demográficas (sexo, idade e estado civil); o segundo, em que estão as variáveis socioeconômicas (renda familiar e escolaridade); o terceiro, que abrange as variáveis comportamentais (nível de estresse, fumo, consumo de bebidas alcoólicas) e de trabalho (satisfação no trabalho); e o quarto nível, no qual estão colocadas as barreiras à prática de atividades físicas no lazer. Os efeitos das variáveis do primeiro nível foram controla- dos entre si; as do segundo nível foram controlados entre elas e para as do primeiro nível; as do terceiro nível foram controladas entre elas e para as dos dois níveis anteriores e a do quarto entre elas e para as dos níveis acima. Para seleção das variáveis que permaneceram no modelo de regressão foi utilizado o processo de seleção para trás e um nível crítico de $\mathrm{p} \leq 0,20$ para permanência, ficando no modelo final todas variáveis que apresentaram valor $\mathrm{p}<0,05$.

A montagem do banco de dados foi efetuada mediante leitura ótica dos questionários [programa Sphynx (Sphynx Software Solutions Inc., Washington DC, Estados Unidos)], a fim de evitar erros de digitação. Para análise estatística, utilizou-se o programa Stata para Windows, versão 10 (Stata Corp., College Station, Estados Unidos). 
O protocolo do estudo foi aprovado pelo Comitê de Ética em Pesquisas da Universidade Federal de Santa Catarina ( $n^{\circ}$. 099/2007), e os trabalhadores assinaram um termo de consentimento livre e esclarecido antes da coleta dos dados.

\section{Resultados}

A amostra final do estudo foi composta de 2.265 trabalhadores industriários, sendo $56,2 \%$ do sexo masculino. A taxa de retorno do questionário foi de $89,6 \%$, sendo representativa de 670.326 trabalhadores da indústria do Estado do Rio Grande do Sul.

A Tabela 1 descreve a população estudada de acordo com as variáveis demográficas, sócioeconômicas, comportamentais e de trabalho. Com relação à idade, o maior extrato da amostra tinha menos de 30 anos (47,6\%), mais da metade era casado/vivia com companheiro $(54,7 \%)$ e a maioria possuía ensino médio completo $(52,2 \%)$. Quase a metade dos entrevistados $(45,6 \%)$ tinha renda familiar compreendida entre $\mathrm{R} \$ 601,00 \mathrm{e}$ $\mathrm{R}$ \$ 1.500,00. Em relação às variáveis estresse e satisfação no trabalho, 88,1\% relataram ter raramente/às vezes sensação de estresse na vida $\mathrm{e}$ mais de 3/5 disseram se sentir bem no trabalho. Aproximadamente 3/4 dos trabalhadores nunca havia fumado e $61,5 \%$ não consumiam bebidas alcoólicas durante a semana habitual.

Observou-se uma prevalência de inatividade física no lazer de 45,6\% (IC95\%: 43,4-47,6) entre os trabalhadores da indústria do Estado do Rio Grande do Sul. As barreiras percebidas mais prevalentes à prática de atividade física foram cansaço 15,1\% (IC95\%: 13,6-16,6), seguido de excesso de trabalho 12,7\% (IC95\%: 11,4-14,1) e obrigações familiares 9,2\% (IC95\%: 8,1-10,1) (Tabela1). Um percentual de $21,3 \%$ dos entrevistados não percebeu dificuldade para realização de atividades físicas no tempo de lazer.

A Tabela 2 mostra a prevalência das barreiras relatadas e sua associação com as variáveis independentes. O sexo feminino esteve associado à barreira obrigações familiares $(\mathrm{p}<0,001)$. A idade esteve inversamente associada com cansaço, obrigações de estudo e falta de dinheiro e apresentou associação em forma de U com falta de vontade e obrigações familiares. No que se refere ao estado civil, indivíduos casados relataram maior prevalência nas barreiras obrigações familiares e falta de vontade, e a barreira obrigações com o estudo foi mais freqüente entre os solteiros. Quanto menor a escolaridade, maior a prevalência de cansaço. Relação direta foi observada entre a mesma e as barreiras clima e obrigações de estudo e em U com falta de dinheiro.
A variável renda familiar se mostrou associada às barreiras cansaço, falta de vontade, falta de dinheiro, obrigações com o estudo e clima. Quanto mais satisfeitos os trabalhadores em seu trabalho menor o relato das barreiras cansaço, excesso de trabalho e falta de dinheiro, mas, para a barreira clima, a associação verificada foi direta. A variável fumo mostrou-se associada às barreiras falta de vontade e obrigações de estudo; já a variável consumo de bebidas alcoólicas esteve associada às barreiras obrigações familiares de forma inversa e falta de dinheiro de forma direta. A variável nível de estresse apresentou-se associada à barreira excesso de trabalho.

A Tabela 3 apresenta os resultados da análise multivariável entre barreiras à prática de atividades físicas e a inatividade física no lazer. Barreiras cansaço $(\mathrm{p}<0,002)$, falta de vontade $(\mathrm{p}<$ $0,001)$, obrigações familiares ( $p<0,001)$, falta de dinheiro ( $\mathrm{p}<0,001)$, obrigações de estudo $(\mathrm{p}<$ $0,001)$, excesso de trabalho $(\mathrm{p}<0,001)$ e clima $(\mathrm{p}=0,02)$ permaneceram associadas à inatividade física no lazer após ajuste para as variáveis sexo, idade, escolaridade, renda, estado civil, consumo de bebidas alcoólicas, fumo, estresse, satisfação no trabalho e demais barreiras. A barreira falta de vontade foi a que apresentou maior efeito sobre o desfecho $(\mathrm{RP}=2,02)$.

\section{Discussão}

Aspecto fundamental a ser destacado no presente estudo é que ele é o primeiro no Brasil a investigar a percepção de barreiras à prática de atividade física no lazer em uma amostra representativa de trabalhadores industriários do Estado do Rio Grande do Sul. Além disso, a alta taxa de retorno dos questionários $(89,6 \%)$ e a padronização dos métodos de coleta de dados devem ser evidenciadas.

Algumas limitações também precisam ser consideradas. $\mathrm{O}$ delineamento transversal utilizado na pesquisa não permite inferir relações de causa-efeito entre barreiras à prática de atividades físicas no lazer e inatividade física no lazer, especialmente pela dificuldade de estabelecer temporalidade. A substituição de trabalhadores selecionados que se negaram a participar, pode resultar em alteração da prevalência de barreiras e nos valores de associação (viés do não respondente).

Analisando a população total do estudo, verificam-se valores elevados de inatividade física no lazer. Comparado à literatura internacional, os dados de inatividade física encontrados $(45,4 \%)$ foram maiores do que os observados em países como a Suécia (32\%) e a Irlanda (35\%) 18 e infe- 
Prevalências das sete barreiras mais reportadas para atividade física no lazer segundo as variáveis independentes. Rio Grande do Sul, Brasil, 2007.

\begin{tabular}{|c|c|c|c|c|c|c|c|}
\hline Variáveis & Cansaço & $\begin{array}{c}\text { Excesso de } \\
\text { trabalho }\end{array}$ & $\begin{array}{l}\text { Obrigações } \\
\text { familiares }\end{array}$ & $\begin{array}{l}\text { Falta de } \\
\text { vontade }\end{array}$ & $\begin{array}{l}\text { Falta de } \\
\text { dinheiro }\end{array}$ & $\begin{array}{l}\text { Obrigações } \\
\text { de estudo }\end{array}$ & Clima \\
\hline \multicolumn{8}{|l|}{ Sexo } \\
\hline Masculino & 14,29 & 13,57 & 5,59 * & 8,62 & 8,06 & 7,42 & 3,75 \\
\hline Feminino & 16,41 & 11,76 & 14,14 & 9,49 & 8,98 & 7,84 & 3,92 \\
\hline \multicolumn{8}{|l|}{ Idade (anos) } \\
\hline$<30$ & $18,09 * \star$ & 11,65 & $6,72 * \star$ & 6,44 * & $8,81 \star \star$ & $12,69 * *$ & 3,79 \\
\hline $30-39$ & 13,06 & 13,84 & 13,69 & 9,95 & 11,04 & 3,58 & 3,73 \\
\hline $40-49$ & 11,78 & 12,57 & 11,26 & 13,35 & 6,28 & 2,62 & 3,40 \\
\hline $50+$ & 10,42 & 15,97 & 3,47 & 11,11 & 1,39 & 2,08 & 5,56 \\
\hline \multicolumn{8}{|l|}{ Estado civil } \\
\hline Solteiro & 15,64 & 12,18 & $5,15 *$ & 7,13 * & 9,01 & 12,08 * & 4,46 \\
\hline Casado/Vive com companheiro & 14,78 & 13,14 & 12,73 & 10,51 & 8,13 & 3,94 & 3,28 \\
\hline \multicolumn{8}{|l|}{ Escolaridade } \\
\hline Fundamental incompleto & 19,30 ** & 12,33 & 10,46 & 10,19 & 7,51 ** & 1,07 ** & 2,68 ** \\
\hline Fundamental completo & 19,15 & 11,97 & 11,44 & 9,31 & 7,71 & 2,13 & 2,39 \\
\hline Médio completo & 13,72 & 13,04 & 8,92 & 8,32 & 10,89 & 10,46 & 4,03 \\
\hline Superior completo & 10,90 & 12,82 & 6,73 & 9,62 & 1,92 & 11,22 & 6,09 \\
\hline \multicolumn{8}{|l|}{ Renda (R\$) } \\
\hline Até 600 & $19,69 * \star$ & 14,17 & 8,14 & $5,77 \star \star$ & $11,81 \star \star$ & $3,41 \star \star$ & 3,15 ** \\
\hline $601-1.500$ & 15,38 & 12,00 & 10,42 & 9,13 & 11,21 & 6,75 & 2,78 \\
\hline $1.501-3.000$ & 13,43 & 13,78 & 9,01 & 9,72 & 4,06 & 10,78 & 4,95 \\
\hline$>3.000$ & 10,89 & 10,89 & 7,66 & 12,10 & 2,82 & 10,89 & 6,95 \\
\hline \multicolumn{8}{|l|}{ Nível de estresse } \\
\hline Raramente/Às vezes & 14,66 & 11,70 * & 9,16 & 8,85 & 8,50 & 7,94 & 4,07 \\
\hline Quase sempre/Sempre & 18,87 & 20,38 & 10,19 & 9,81 & 8,68 & 5,66 & 1,89 \\
\hline \multicolumn{8}{|l|}{ Satisfação no trabalho } \\
\hline Muito Bem & $10,75 * \star$ & $8,64 * \star$ & 9,02 & 9,60 & $5,37 \star \star$ & 7,68 & $6,14 * *$ \\
\hline Bem & 15,70 & 12,87 & 9,00 & 9,30 & 9,38 & 7,59 & 3,50 \\
\hline Mais ou menos & 19,82 & 18,05 & 10,95 & 6,51 & 9,47 & 7,69 & 1,78 \\
\hline Mal & 13,64 & 13,64 & 9,09 & 9,09 & 18,18 & 13,64 & 0,00 \\
\hline Muito mal & 25,00 & 25,00 & 0,00 & 25,00 & 0,00 & 0,00 & 0.00 \\
\hline \multicolumn{8}{|l|}{ Fumo } \\
\hline Nunca fumou & 14,36 & 12,35 & 9,59 & 8,84 * & 7,71 & 9,22 * & 3,76 \\
\hline Ex-fumante & 17,71 & 12,86 & 9,14 & 6,00 & 11,14 & 4,00 & 4,00 \\
\hline Atual fumante & 16,67 & 14,89 & 7,80 & 13,48 & 9,57 & 2,84 & 3,90 \\
\hline \multicolumn{8}{|l|}{ Bebidas alcoólicas (doses) } \\
\hline Nenhuma & 15,40 & 12,46 & $11,07 * \star$ & 8,43 & 7,84 ** & 7,84 & 3,15 \\
\hline $1-7$ & 15,06 & 13,80 & 6,58 & 9,62 & 8,99 & 7,59 & 4,81 \\
\hline $8-14$ & 10,20 & 6,12 & 6,12 & 14,29 & 20,41 & 6,12 & 4,08 \\
\hline 15 ou mais & 23,08 & 15,38 & 7,69 & 0,00 & 0,00 & 0,00 & 7,69 \\
\hline
\end{tabular}

* Qui-quadrado de Pearson para heterogeneidade, $p<0,05$;

** Teste de qui-quadrado de Pearson para tendência linear, $p<0,05$. 
Análise bruta e ajustada da associação entre barreiras percebidas e a inatividade física no lazer.

\begin{tabular}{lccc}
\hline Barreiras & Bruta (IC95\%) & Ajustada * (IC95\%) & Valor de p \\
\hline Cansaço & $0,99(0,87-1,12)$ & $1,28(1,10-1,50)$ & 0,002 \\
Excesso de trabalho & $1,01(0,88-1,16)$ & $1,31(1,12-1,55)$ & 0,001 \\
Obrigações familiares & $1,56(1,40-1,73)$ & $1,74(1,51-2,01)$ & $<0,001$ \\
Falta de vontade & $1,68(1,52-1,86)$ & $2,02(1,77-2,31)$ & $<0,001$ \\
Falta de dinheiro & $1,16(1,00-1,35)$ & $1,53(1,29-1,81)$ & $<0,001$ \\
Obrigações de estudo & $1,06(0,90-1,25)$ & $1,56(1,29-1,88)$ & $<0,001$ \\
Clima desfavorável & $0,40(0,26-0,63)$ & $0,59(0,37-0,93)$ & 0,02 \\
\hline
\end{tabular}

* Ajustada para sexo, idade, situação conjugal, consumo de bebidas alcoólicas, fumo, estresse, satisfação no trabalho e demais barreiras. Todas variáveis de ajuste apresentaram-se associadas ao desfecho $(p \leq 0,05)$.

riores aos de Portugal $(87,8 \%)$ 19. Contudo, inquéritos nacionais encontraram prevalências muito semelhantes às de nosso estudo 20,21 como, por exemplo, em Florianópolis, onde $46,2 \%$ dos trabalhadores da indústria foram considerados inativos no lazer 22 .

Os resultados do presente estudo mostraram que sentir-se cansado foi a barreira mais prevalente $(15,1 \%)$, seguida de excesso de trabalho $(12,7 \%)$, entre trabalhadores da indústria. Similarmente, estudos brasileiro 10 e europeu 13 constataram altas prevalências dessas barreiras em adultos. Cabe salientar que ambas as barreiras podem refletir a falta de motivação para o envolvimento na atividade física. Fatores motivacionais têm demonstrado forte associação com o nível de atividade física 8,23 .

Possível hipótese é que indivíduos que realizam atividades ocupacionais mais intensas, como as que exigem levantamento de peso, posição viciosa, deslocamento constante, entre outras, podem estar sujeitos à fadiga e, em alguns casos, à dor muscular, o que pode levar, conseqüentemente, à desmotivação para a realização de atividades físicas no seu tempo livre. Atualmente, homens e, especialmente, mulheres realizam dupla jornada de trabalho, combinando a realização de tarefas domésticas e ocupacionais que os expõem a cargas ergonômicas, particularmente repetitividade, posição viciosa e trabalho em grande velocidade 24 .

A barreira falta de vontade foi a que apresentou maior efeito sobre a inatividade física, concordando com os resultados descritos em outros estudos 9,12, sendo essa variável amplamente relatada em estudos sobre barreiras à prática de atividades físicas 10,12.

O presente estudo também identificou características dos trabalhadores que tiveram maior probabilidade de perceberem barreiras particulares à prática de atividade física no lazer (barreiras específicas pertencentes a um determinado grupo de indivíduos com características sócioeconômicas distintas diferem na percepção de barreiras à prática de atividades físicas no lazer). Algumas barreiras são mais prevalentes em trabalhadores com baixa escolaridade e menor nível socioeconômico, confirmando a hipótese de que indivíduos com alta renda têm maior probabilidade de ser ativos no lazer em virtude de maior motivação e acesso a recursos para a realização dessas práticas 25 , resultados consistentemente reportados em estudos na Austrália e na União Européia 9,13.

Ao analisar as prevalências das barreiras para atividade física segundo as variáveis independentes, observou-se que a barreira obrigações familiares esteve associada ao sexo feminino e a indivíduos casados. Booth et al. ${ }^{9}$ demonstraram que a responsabilidade de cuidar dos filhos também é considerada uma importante barreira à prática de atividades físicas para indivíduos australianos. Estudo realizado na cidade de Toronto, Canadá 26 , reforça a diferença na percepção de barreiras com relação ao sexo.

O grande número de barreiras associadas ao sexo feminino possivelmente deve-se a aspectos de ordem socioculturais. Desde cedo, homens são estimulados a praticar atividades físicas, como jogar futebol, pedalar, correr. Por sua vez, indivíduos do sexo feminino são estimulados a praticar atividades menos intensas 27 .

Ainda na análise bruta, encontrou-se que quanto mais satisfeitos os trabalhadores estão com seu trabalho, menor o relato das barreiras cansaço, excesso de trabalho e falta de dinheiro. Esta variável pode, na verdade, ser marcador do nível socioeconômico e escolaridade. 
Percebe-se que as prevalências da barreira falta de dinheiro são altas em países em desenvolvimento 10 , por outro lado, esses resultados diferem em países desenvolvidos 9. Em países ricos, a falta de tempo é muito reportada como barreira e pode ser fomentada pela falta de motivação ${ }^{13}$. Em contrapartida, a dificuldade financeira é pouco relatada 10 . Já em países em desenvolvimento, com realidades opostas, outros tipos de limitações, entre elas a falta de dinheiro, são apresentados frequentemente, e a prevalência dessas barreiras são maiores, como já citado anteriormente.

A barreira clima, em trabalhadores da indústria, evidencia uma realidade inversa àquela que vem sendo relatada por outros pesquisadores, que é a dificuldade de praticar atividades físicas ao ar livre em função da instabilidade do clima em determinadas épocas do ano. Estudos relatam que condições climáticas inadequadas têm sido associadas negativamente com o nível de atividade física 5,28. Apesar disso, os dados dos trabalhadores mostram que o clima foi fator de proteção à inatividade física.

Com relação à barreira obrigações de estudo, constatou-se que ela pode estar relacionada à falta de tempo 26 , visto que o maior extrato da amostra foi composto por trabalhadores com idade inferior ou igual a 30 anos, que possivelmente dedicam o seu tempo ao trabalho e aos estudos.
Concluindo, este estudo além de verificar uma elevada prevalência de inatividade física no lazer, identificou que as barreiras mais prevalentes à prática de atividades físicas no lazer em trabalhadores da indústria no Estado do Rio Grande do Sul foram cansaço, excesso de trabalho e obrigações familiares. Também constatou que, além das barreiras acima citadas, a falta de vontade, falta de dinheiro, obrigações de estudo e clima estiveram associados à inatividade física no lazer nesses indivíduos.

Mesmo verificando associação entre a inatividade física no lazer e as barreiras citadas anteriormente, é importante salientar que variáveis individuais são insuficientes para entender a complexidade de um fenômeno como a prática de atividade física. As barreiras estão inseridas num contexto que inclui aspectos culturais, econômicos, políticos e geográficos.

Ações educativas sobre a importância da prática regular de atividades físicas bem como a criação e melhoria de espaços a essas práticas, são pontos cruciais a serem focados por políticas públicas voltadas à saúde e à qualidade de vida. No que se refere às empresas, disponibilidade de locais destinados ao lazer e deslocamento ativo, bem como a melhoria da distribuição da carga de trabalho diária podem melhorar a qualidade de vida dos trabalhadores e aumento da produtividade.

\section{Resumo}

Identificar as principais barreiras percebidas e verificar os fatores associados à inatividade física no lazer em trabalhadores industriários do Estado do Rio Grande do Sul, Brasil. Estudo transversal com análise secundária de dados, incluindo 2.265 trabalhadores. Treze barreiras foram investigadas: cansaço, clima, excesso de trabalho, falta de vontade, obrigações de estudo, obrigações familiares, distância até o local da prática, falta de habilidade motora, falta de condições físicas, falta de instalações, falta de dinheiro e condições de segurança, além da opção outra. A prevalência de inatividade física no lazer entre os trabalhadores foi $45,4 \%$, e as barreiras mais prevalentes foram cansaço (15,1\%), excesso de trabalho (12,7\%) e obrigações familiares $(9,2 \%)$. Cansaço, excesso de trabalho, obrigações familiares, falta de vontade, falta de dinheiro, obrigações de estudo e clima estiveram associados à inatividade física no lazer. Políticas públicas focadas em macroestratégias de intervenção podem ajudar esses trabalhadores a superar tais barreiras e incorporarem um estilo de vida ativo.

Atividade Motora; Atividades de Lazer; Categorias de Trabalhadores 


\section{Colaboradores}

S. G. Silva participou de todas as etapas da pesquisa e escreveu o artigo. M. C. Silva realizou a análise dos dados e redação final do artigo. M.V. Nahas foi responsável pela coordenação do trabalho de campo e participou da redação final do artigo. S. L. Viana participou da redação final do artigo.

\section{Referências}

1. Cox MH, Miles DS. Workplace active living and total quality management: a paradigm for a new corporate culture. In: Quiney HA, Gauvin L, Wall AET, editors. Toward active living. Champaign: Human Kinetics; 1994. p. 179-86.

2. Gallassi MC. Medicina do trabalho: programa de saúde de controle médico de saúde ocupacional. São Paulo: Editora Atlas; 1999.

3. Sallis JF, Nader P, Broyles S, Berry C, Elder J, Mackenzie T, et al. Correlates of physical activity at home in Mexican-American and Anglo-American preschool children. Health Psychol 1993; 12:390-8.

4. Sallis JF, Conway TL, Prochaska JJ, McKenzie TL, Marshall SJ, Brown M. The association of school environments with youth physical activity. Am J Public Health 2001; 91:618-20.

5. Sallis JF, Owen N. Physical activity \& behavioral medicine. London: Sage Publications; 1999.

6. Hallal PC, Victora CG, Wells JCK, Lima RC. Physical inactivity: prevalence and associated variables in Brazilian adults. Med Sci Sports Exerc 2003; 38:1894-900.
7. Monteiro CA, CondeWL, Matsudo SM, MatsudoVR, Bonseñor IM, Lotufo PA. A descriptive epidemiology of leisure-time physical activity in Brazil, 19961997. Rev Panam Salud Pública 2003; 14:246-54.

8. Trost SG, Owen N, Bauman AE, Sallis JF, Brown W. Correlates of adults participation in physical activity: review and update. Med Sci Sports Exerc 2002; 34:1996-2001.

9. Booth ML, Bauman A, Owen N, Gore CJ. Physical activity preferences, preferred sources of assistance, and perceived barriers to increased activity among physically inactive Australians. Prev Med 1997; 26:131-7.

10. Reichert FF, Barros AJD, Domingues MR, Hallal PRC. The role of perceived personal barriers to engagement in leisure-time physical activity. Am J Public Health 2007; 97:515-9.

11. Oman R. Barriers to leisure-time physical activity in an inner city, minority population. Res Q Exerc Sport 1998; Suppl:A-44. 
12. Bowles HR, Morrow Jr. JR, Leonard BL, Hawkins M, Couzelis PM. The association between physical activity behavior and commonly reported barriers in a worksite population. Res Q Exerc Sport 2002; 73:464-70.

13. Zunft HJ, Friebe D, Seppelt B, Widhalm K, Winter AR, Almeida MDV, et al. Perceived benefits and barriers to physical activity in a nationally representative sample in the European Union. Public Health Nutr 1999; 2:153-60.

14. Martins CO, Michels G. Programas de promoção da saúde do trabalhador: exemplos de sucesso. Rev Bras Cineantropom Desempenho Hum 2003; 5:85-90.

15. Nahas MV, Barros MV, Oliveira ES, Aguiar FS. Estilo de vida e hábitos de lazer dos trabalhadores das indústrias brasileiras: relatório geral. Brasília: Serviço Social da Indústria, Departamento Nacional; 2009.

16. Luiz RR, Magnanini MF. A lógica da determinação do tamanho da amostra em investigações epidemiológicas. Cad Saúde Colet (Rio J.) 2000; 8:9-28.

17. Barros MVG. Atividades físicas no lazer e outros comportamentos relacionados à saúde dos trabalhadores da indústria no Estado de Santa Catarina, Brasil [Dissertação de Mestrado]. Florianópolis: Centro de Desportos, Universidade Federal de Santa Catarina; 1999.

18. Vouri IM. Health benefits of physical activity with special reference to interaction with diet. Public Health Nutr 2001; 4:517-28.

19. Camões M, Lopes C. Fatores associados à atividade física na população portuguesa. Rev Saúde Pública 2008; 42:208-16.

20. Salles-Costa R, Heilborn ML, Werneck GL, Faerstein E, Lopes CS. Gênero e prática de atividade física de lazer. Cad Saúde Pública 2003; 19 Suppl 2:S325-33.
21. Monteiro CA, Moura EC, Jaime PC, Lucca A, Florindo AA, Figueiredo IC, et al. Monitoramento de fatores de risco para doenças crônicas por entrevistas telefônicas. Rev Saúde Pública 2005; 39: 47-57.

22. Barros MV, Nahas MV. Comportamentos de risco, auto-avaliação do nível de saúde e percepção de estresse entre trabalhadores da indústria. Rev Saúde Pública 2001; 35:554-63.

23. Sherwood NE, Jeffery RW. The behavioral determinants of exercise: implications for physical activity interventions. Annu Rev Nutr 2000; 20:21-44.

24. Silva MC, Fassa AG, Valle NCJ. Dor lombar crônica em uma população adulta do Sul do Brasil: prevalência e fatores associados. Cad Saúde Pública 2004; 20:377-85.

25. Dias-da-Costa JS, Hallal PC, Wells JCK, Daltoé T, Fuchs SC, Menezes AMB, et al. Epidemiology of leisure-time physical activity: a population-based study in southern Brazil. Cad Saúde Pública 2005; 21:275-82.

26. Allison KR, Dwyer JJ, Makin S. Perceived barriers to physical activity among high school students. Prev Med 1999; 28:608-15.

27. Pate RR, Freedson PS, Sallis JF, Taylor WC, Sirard J, Trost SG, et al. Compliance with physical activity guidelines: prevalence in a population of children in youth. Ann Epidemiol 2002; 12:303-8.

28. Chan CB, Ryan DA, Tudor-Locke C. Relationship between objective measures of physical activity and weather: a longitudinal study. Int J Behav Nutr Phys Act 2006; 3:21.

Recebido em 14/Abr/2010

Versão final reapresentada em 14/Nov/2010

Aprovado em 24/Nov/2010 